\title{
Space Dependent Mean Field Approximation Modelling
}

\author{
M. R. Dudek · J. N. Grima · R. Cauchi - C. Zerafa • \\ R. Gatt • B. Zapotoczny
}

Received: 17 November 2013 / Accepted: 30 January 2014 / Published online: 13 February 2014

C) Springer Science+Business Media New York 2014

\begin{abstract}
It is shown that the self-consistency condition which is the basic equation for calculating the mean-field order parameter of any mean-field model Hamiltonian can be replaced by the standard Metropolis Monte Carlo scheme. The advantage of this method is its ease of implementation for both the homogeneous mean-field order parameter and the heterogeneous one. To be specific, the mean-field version of the Ising model spin system is discussed in detail and the resulting magnetization is the same as in the case of solving the respective mean-field self-consistency equation. In addition, it is shown that if a high temperature phase of such system is quenched below critical temperature then the mean field experienced by spins develops into a network of domains in analogous way as it happens with the spins in the case of the exact many-body Hamiltonian system and the coarsening processes start to take place. To show that the introduced Metropolis Monte Carlo method works also in case of the continuous variables the order parameter for the Maier-Saupe model for nematic liquid crystals has been calculated.
\end{abstract}

Keywords Mean field approximation · Magnetic domains · Coarsening · Monte Carlo method

\section{Introduction}

The history of the mean-field theory goes back to Van der Waals's works and to 1907 when Weiss [1] used the idea of the molecular field to explain the magnetic ordering in ferromagnets

\footnotetext{
M. R. Dudek $(\varangle) \cdot$ B. Zapotoczny

Institute of Physics, University of Zielona Góra, ul. Szafrana 4a, 65-069 Zielona Góra, Poland

e-mail: M.Dudek@if.uz.zgora.pl
}

\section{J. N. Grima}

Metamaterials Unit, Faculty of Science, University of Malta, Msida 2080, Malta

J. N. Grima · R. Cauchi · C. Zerafa · R. Gatt

Department of Chemistry, Faculty of Science, University of Malta, Msida 2080, Malta 
(see the recent review on mean-field theory by Kadanoff [2]). A generalization of the Weiss mean-field theory by Landau [3] with an expansion of the free energy in an order parameter gave a new modern understanding of the phase transitions through the symmetry breaking idea $[2,4]$. This paper addresses the mean field theory with spatially varying order parameters such as Ginzburg-Landau theory [5], mean-field model for spinodal decomposition [6] or spatially modulated systems [7]. Note that the mean field approximation is popular both in classical and quantum statistical model description and still the new methods are developing to improve the approximation, e.g. [8,9]. The mean-field approach is usually used when it is computationally very hard to solve a particular statistical physics problem with the help of other methods.

To be specific, let us consider a 2-body interacting system of magnetic moments $s_{i j}$ located in the sites of a $L \times L$ square lattice for which the Hamiltonian is the nearest-neighbour Ising Model Hamiltonian [4]

$$
H_{\text {exact }}=-J \sum_{\langle i j, k l\rangle} s_{i j} s_{k l}-h \sum_{i, j=1}^{L} s_{i j}
$$

where Ising spins $s_{i j}= \pm 1$, the angular brackets denote summation over the nearestneighbour lattice pairs, $h$ is the external magnetic field, $J$ is the exchange integral. Following the Weiss mean field approximation with variation in space, the exact Hamiltonian in Eq. (1) can be converted into a mean field one-body Hamiltonian in the form

$$
H_{\mathrm{MFA}}=J \sum_{\langle i j, k l\rangle} m_{i j} m_{k l}-J \sum_{\langle i j, k l\rangle} m_{k l} s_{i j}-h \sum_{i, j=1}^{L} s_{i j},
$$

where each spin $s_{i j}$ experiences an effective magnetic field

$$
H_{i j}^{\mathrm{eff}}=h+J\left(m_{i-1, j}+m_{i+1, j}+m_{i, j-1}+m_{i, j+1}\right)
$$

with $m_{i j}=\left\langle s_{i j}\right\rangle$ being the mean field parameters. The values of $m_{i j}$ which minimize the system free energy $F\left(k_{B} T,\left\{m_{i j}\right\}_{1,1}^{L, L}, h\right)$, where $k_{B}$ represents the Boltzmann constant and $T$ is temperature, are obtained from the self-consistency condition $\delta F\left(k_{B} T,\left\{m_{i j}\right\}_{1,1}^{L, L}, h\right)=0$ for finding free energy extremes with respect to $m_{i j}$ which lead to a set of $N=L^{2}$ nonlinear equations

$$
m_{i j}=m_{i j}\left(J / k_{B} T, m_{11}, m_{12}, \ldots m_{L L}, h\right) ; i, j=1, \ldots, L
$$

Finding the equations Eq. (4) and their solutions $m_{i j}$ at lattice sites $(i, j)$ is the well known traditional method for determining the local mean-field parameters. In the particular case of the uniform solution, where $m_{i j}=m$, the equilibrium magnetization $m$ reads as:

$$
m=\tanh \left(\frac{z J m+h}{k_{B} T}\right),
$$

where $z$ denotes the number of nearest neighbours.

The novelty of our method of calculating the local mean-field parameters is that we performed a Metropolis Monte Carlo computer experiment for $L_{s}$ replicas of $N$ Ising spins $s_{i j}(i, j=1, \ldots, L)$ described by the one-body Hamiltonian in Eq. (2) instead of deriving and solving the set of nonlinear equations in Eq. (4). The method is justified by the fact that the Metropolis algorithm satisfies the principle of detailed balance [10] and therefore the resulting mean field kinetics leads to the state of thermodynamic equlibrium. The proposed 
numeric scheme is similar to the Monte Carlo mean field theory introduced by Netz and Berker [11] and applied to frustrated spin systems. These two methods are equivalent for homogeneous systems. Note that finding solutions for a large system of nonlinear equations in Eq. (4) with given complex boundary conditions can be very difficult using standard numerical methods. In addition, some statistical models which are described with the help of the continuous variables will be represented by a set of nonlinear integral equations for which in general can be a lot harder to find a solution than in the case of Eqs. (4) or (5) for Ising spins. An example is the well-known mean-field Maier-Saupe model [12] for nematic liquid crystals with the following Hamiltonian:

$$
H_{\mathrm{MFA}}=-\varepsilon\left\langle P_{2}\right\rangle P_{2}(\cos (\theta)),
$$

where $\theta$ is the angle of a liquid crystal molecule with respect to the nematic director $n$, $P_{2}(\cos (\theta))=\frac{1}{2}\left(3 \cos ^{2}(\theta)-1\right)$ is the second-order Legendre polynomial and $\varepsilon$ is the interaction strength between liquid crystal molecules. In the case of a uniform mean-field parameter (the analogue of Eq. 5) the value of the mean field parameter $\left\langle P_{2}\right\rangle$ is calculated from the self-consistency equation which takes the following form [13]:

$$
\left\langle P_{2}\right\rangle=\int_{-1}^{1} d(\cos (\theta)) f(\theta) P_{2}(\cos (\theta)),
$$

where $f(\theta)=\frac{1}{Z} \mathrm{e}^{-H_{\mathrm{MFA}} / k_{B} T}$ represents the distribution function for $\theta$ and $Z$ is the respective partition function [13]. The integration on the right-hand side of Eq. (7) can be found with the help of the Dawson integral [14]. The calculation of $\left\langle P_{2}\right\rangle$ with space variation (the analogue of Eq. 4) is much more difficult. The method introduced by us replaces the self-consistency equation Eq. (7) by a simple Monte Carlo scheme.

\section{Mean Field Metropolis Monte Carlo Algorithm}

To be specific, we consider a mean field version of the two-dimensional Ising Model Hamiltonian (Eq. 2) which is defined on a square $L \times L$ lattice, e.g. with the periodic boundary conditions imposed. In the model under consideration, each lattice site is occupied by a finite number $L_{s}$ of replicas of Ising spin $\left\{s_{k}\right\}_{1}^{L_{s}}$. The initial mean field magnetization $m_{i j}$ at site $(i, j)(i, j=1, \ldots, L)$ is defined as an average over all spin replicas at this site, i.e.

$$
m_{i j}=\frac{1}{L_{s}} \sum_{k=1}^{L_{s}}\left(s_{k}\right)_{i j} .
$$

The algorithm leading to obtain the equilibrium values of $m_{i j}$ is based on the Metropolis Monte Carlo algorithm and it is the following:

1. Initial set up: for each lattice site $(i, j)$ choose an initial set of Ising spin replicas $\left(\left\{s_{k}\right\}_{1}^{L_{s}}\right)_{i j}$ populating the sites and calculate the corresponding site magnetization $m_{i j}$.

2. Perform a given number of Monte Carlo steps:

(i) Choose at random one spin $s$ from among $L_{s} L^{2}$ spins populating all lattice sites (e.g. one of $L_{s}$ spins at a site $(i, j)$ has been chosen), calculate energy $E(s)$ (Eq. 2)

(ii) Try to change the value of $s$ to $s^{\prime}=-s$. If $\Delta E=E\left(s^{\prime}\right)-E(s)<0$, then accept the change, otherwise accept the change with probability $\min \left(1, \exp \left(-\Delta E / k_{B} T\right)\right)$.

(iii) Calculate the new value of $m_{i j}$ (Eq. 8) if the change from $s$ to $s^{\prime}$ is successful. 


\section{Discussion of the Results}

In the presented mean field Metropolis Monte Carlo algorithm the initial values of $m_{i j}$ at each site $(i, j)$ are calculated from the initial spin configuration $\left(\left\{s_{k}\right\}_{1}^{L_{s}}\right)_{i j}$ with the help of Eq. (8). In each case after application of the algorithm, they tend to their thermodynamic equilibrium values with the help of the spin replicas populating each lattice site which increase or decrease $m_{i j}$ in each Monte Carlo step or they leave it unchanged. The approaching of the equilibrium value by $m_{i j}$ is evident in Fig. 1, which shows the dependence of the absolute value of the mean field magnetization $m=\frac{1}{N} \sum_{i, j=1}^{L} m_{i j}$ on temperature $T$ for different spin system sizes $L=5$ and $L=40$. In Fig. 1, all spins initially were set to $\left(\left\{s_{k}\right\}_{1}^{L_{s}}\right)_{i j}=1$. The number of MC steps is equal to 1,000 . Note that even small system size $L=5$ well approximates the exact mean field magnetization (Eq. 5). To show that the substitution of the self-consistency condition for the mean-field order parameter is not restricted only to the Ising model spin system there have been presented in Fig. 2 the analogous results for the mean-field order parameter $\left\langle P_{2}\right\rangle$ in the Maier-Saupe model with the Hamiltonian in Eq. (6).

Another advantage of the above algorithm is that ease of its implementation remains the same regardless of the shape of the boundary conditions. The cpu time which is necessary for execution of the algorithm on a square lattice grows quadratically with the lattice size $L$, i.e. $\tau \sim L^{2}$.

It is well known that the quench of the high-temperature phase of the system to its low temperature phase $\left(\mathrm{T}<\mathrm{T}_{\mathrm{c}}\right.$ ) always leads to competing domains and their average size grows with time as $t^{\alpha}$, where $\alpha$ depends on the type of dynamics (without conserved order parameter) that dominates the system evolution [15,16]. In case of Ising spin systems (Eq. 1) in spatial dimensions $d>1$ the exponent $\alpha=1 / 2$. One of the first qualitative explanations of coarsening processes has been proposed by Lifshitz and Slyozov [17].

In our mean field Monte Carlo modelling, the kinetics of approaching the thermodynamic equilibrium by the local mean field magnetization results with the domain-like structures

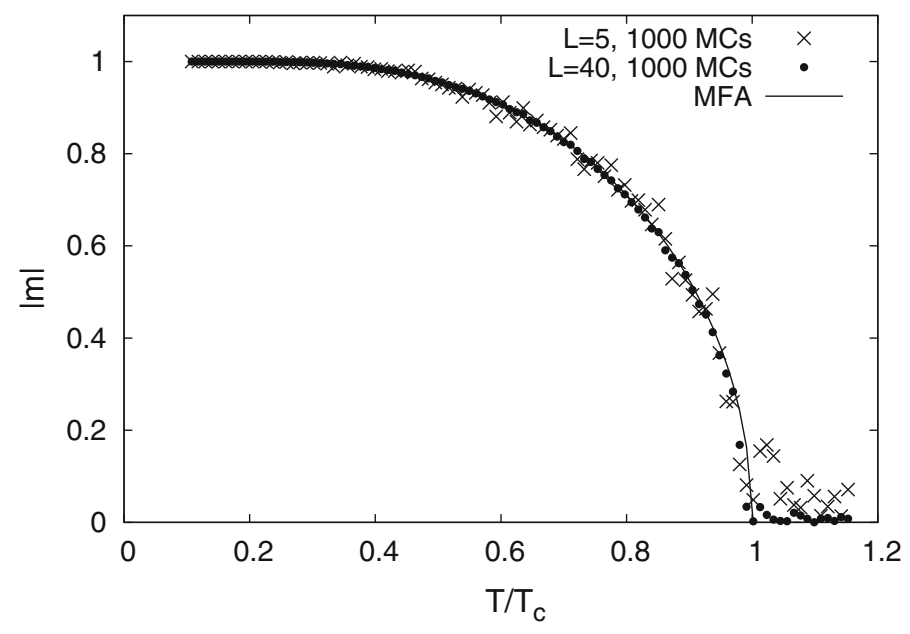

Fig. 1 Dependence of the absolute value of magnetization $|m|$ on temperature for 2D nearest neighbour Ising model in mean field approximation (Eq. 2) when $h=0$. The results of computer simulation of the spin system on square lattice size $L \times L(L=5$ and $L=40)$ with periodic boundary conditions and $t=1,000 \mathrm{MC}$ steps have been presented as well as the mean field approximation expression for magnetization in Eq. (5) 


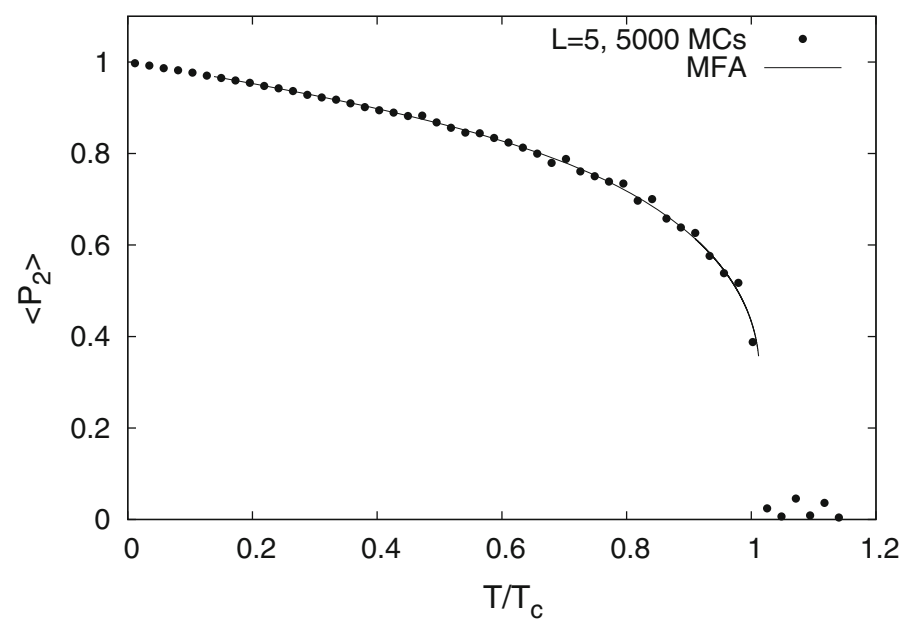

Fig. 2 Dependence of $\left\langle P_{2}\right\rangle$ on temperature for the Maier-Saupe model [12] for nematic liquid crystals. Mean field approximation result from the self-consistency equation (Eq. 7) is compared with the results of the mean-field Monte Carlo simulation. Some parameters: square lattice $L \times L(L=5)$ with periodic boundary conditions, $t=1,000 \mathrm{MC}$ steps, $\varepsilon=0.17$

(areas with the same sign of $m_{i j}$ ) which resemble the ones known for spins. The mean field magnetizations $m_{i j}(\mathrm{i}, \mathrm{j}=1, \mathrm{~L})$ which initially have randomly chosen values undergo the coarsening processes below $\mathrm{T}_{\mathrm{c}}$ exhibiting competing domain-like structures as in Fig. 3. In Fig. 4, the dependence of the gyration radius $R_{\mathrm{g}}=\sqrt{\frac{1}{2 N_{d}^{2}} \sum_{q, q^{\prime}}\left(\vec{r}_{q}-\vec{r}_{q^{\prime}}\right)^{2}}$ (root mean square distance between $N_{d}$ lattice sites belonging to the same domain) on time has been plotted and it suggests $t^{1 / 2}$ power law of the domains growth, the same as observed in other systems with the nonconserved scalar order parameter.

Note that the initial time dependence of $R_{\mathrm{g}}$ is dominated by the small values of the initially chosen $m_{i j}$ and this is the reason that at higher temperatures the range of the scaling region $\left(t^{1 / 2}\right)$ is shifted to later times. On the other hand, the saturation of all the curves in a later time is a finite size effect on the domains growth.

Before we conclude, it is important to note that the idea of the mean field parameter with variation on space can be useful for modelling systems with different kinds of degrees of freedom where some of them is considered in a mean-field approximation, e.g. the molecular dynamics method describing the oscillations of magnetic atoms of a magnet can be combined with the mean field Metropolis Monte Carlo method for system magnetization calculation. In this case, for each time moment $\Delta t$ a given number of Monte Carlo steps is performed to calculate local values of $m_{i j}$. For example, Fig. 5 shows the results that may be obtained on using such hybrid methods where the mean-field magnetization oscillations of a twodimensional magnet have been plotted as a function of time after an external force had been applied to its edges resulting in stretching or compression of the magnet in the longitudinal direction ( $x$-direction). In this case, magnet is represented by a bead-spring model with the Ising spins local mean-field magnetizations $m\left(\vec{r}_{i}\right)$ located in the beads at position $\vec{r}_{i}$. The beads constitute the sites of a square lattice and the bonds represent the springs. The exchange integral $J_{i j}$ is ferromagnetic and it decays as $r_{i j}^{-3}$ with the distance $r_{i j}$ between $\vec{r}_{i}$ and $\vec{r}_{j}$. The corresponding magnetic force is considered to be attractive and its strength $\left|F_{M}\right|=$ $J\left|m\left(\vec{r}_{i}\right) m\left(\vec{r}_{j}\right)\right| / r_{i j}^{3}$. The attractive magnetic interaction between the nearest-neighbour beads is completed by the harmonic bond interations and the Lennard-Jones bond interaction. 
Fig. 3 Two-dimensional maps of the mean field magnetization $m_{i j}$ for the spin system with

Hamiltonian in Eq. (2), where the sites $(i, j)$ represent $y$-coordinate and $x$-coordinate, respectively, are shown after $t=10 \mathrm{MC}$ steps, $t=50 \mathrm{MC}$ steps and $t=250$

MC steps. The palette with color gradients on the right side shows the range of values which takes $m_{i j}$. The periodic boundary conditions have been chosen. Temperature $T=0.65 T_{C}$
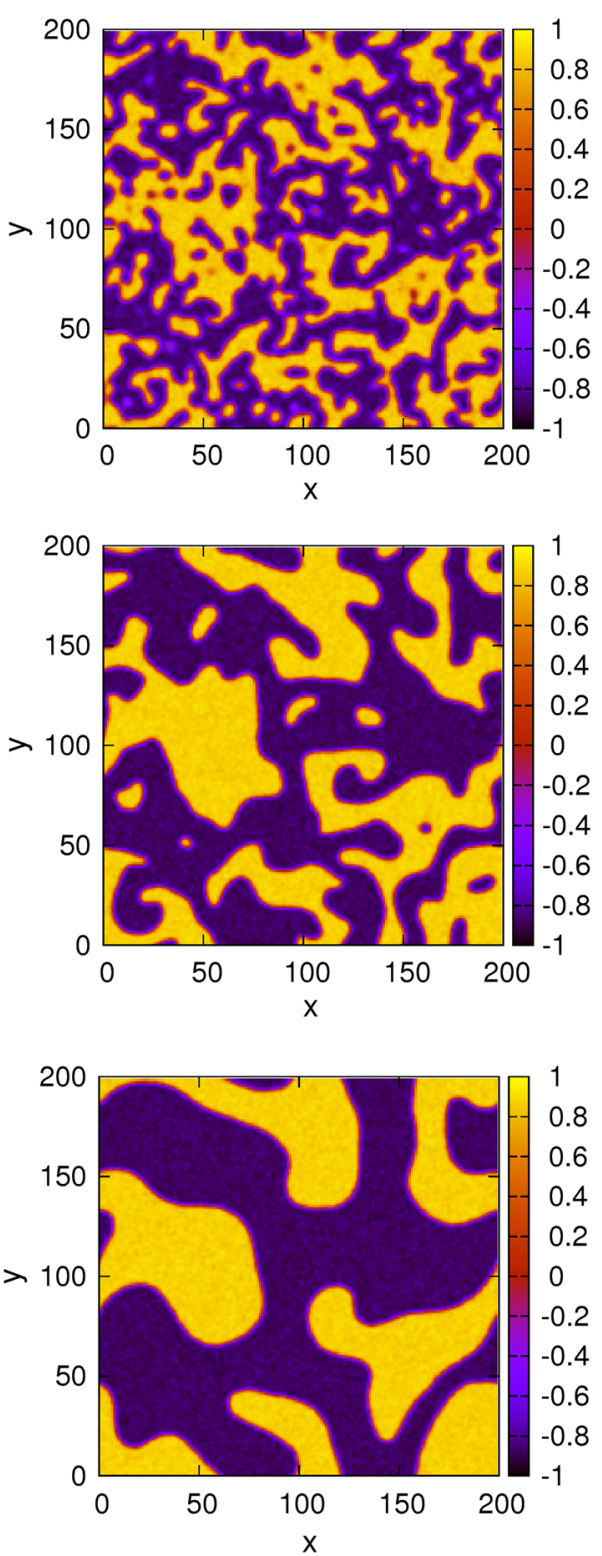

The non-bonded interactions have not been considered. The Nosé-Hoover thermostat $[18,19]$ with the leap-frog molecular dynamics scheme has been used to describe motion of magnetic atoms. It is obviously beyond the scope of this work to give full details as we simply want to illustrate the possibility to use mean-field magnetization for modelling magnetic-elastic coupling as it takes place in Fig. 5.

Similarly, it is obvious that what is discussed here is just one example where the presented extended version of mean field approximation to include space dependent characteristics may be used, and that this improved model can be very useful in various other applications. The results presented in Fig. 2 suggest that the mean-field Metropolis Monte Carlo method 


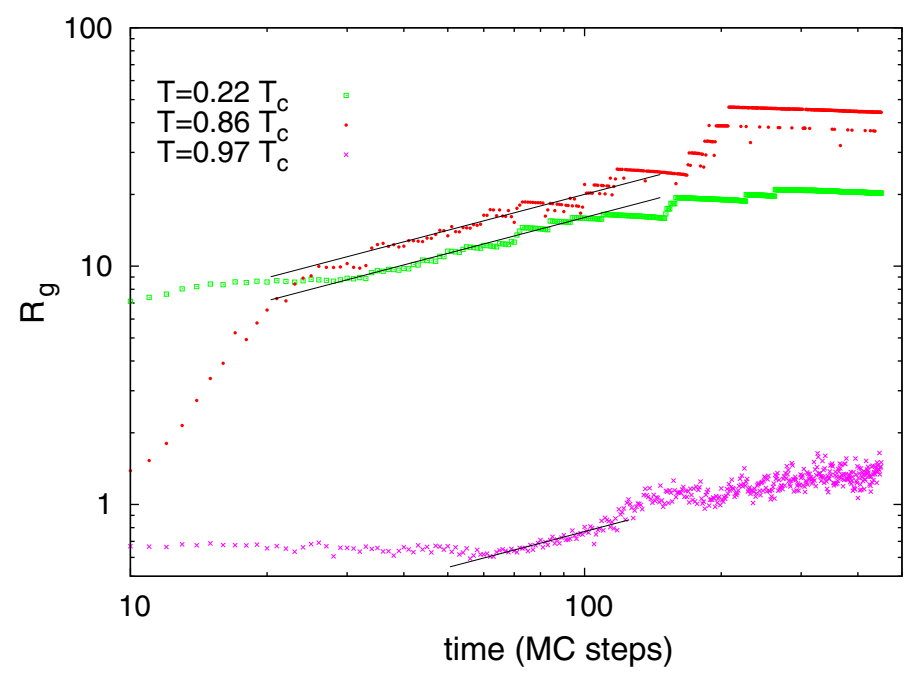

Fig. 4 Dependence of the gyration radius $R_{\mathrm{g}}$ on time for $200 \times 200$ square lattice Ising model in space dependent mean field approximation. The straight lines are guides for eyes only and they suggest $t^{1 / 2}$ power law

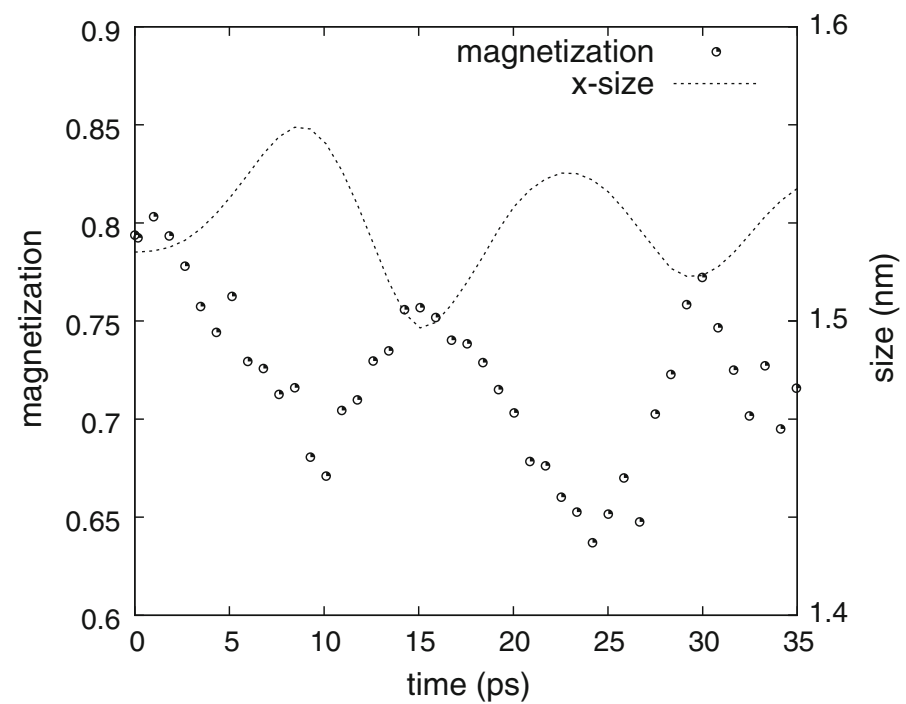

Fig. 5 Oscillations of the mean-field magnetization resulting from the subsequent longitudinal ( $x$-direction) stretching and compressing of the system consisting of $10 \times 10$ beads Some other parameters: $T=0.65 T_{c}$, equilibrium lattice constant $a=0.17 \mathrm{~nm}$, Number of MC steps per $\Delta t$ is equal to 50

introduced by us can be easily extended to non-magnetic systems. One of the advantages of the current approach with respect to straight Monte Carlo simulations of the underlying Hamiltonian can be its application to describe deformation behaviour of some materials, e.g. polymeric materials or foams, in order to obtain significantly shorter duration of the computer simulation. 


\section{Conclusion}

It has been shown the possibility of introducing mean-field Metropolis Monte Carlo scheme for a mean-field model instead of solving the respective self-consistency equation. The strength of the presented method is the ease of its implementation in computer simulations even in the case of the mean field parameter with variation on space. It has been shown that the growth of the mean field domains follows the same power law as it is observed for the domains in the exact models. It is important feature for these applications where some of the system components need not be represented exactly.

Acknowledgments We thank professor Dietrich Stauffer for his comments on the mean-field Metropolis Monte Carlo algorithm as well as his suggestions concerning the paper. C. Zerafa and R. Cauchi acknowledge the support of the Strategic Educational Pathways Scholarship Scheme (Malta). These STEPS scholarships are part-financed by the European Union European Social Fund. B. Zapotoczny thanks for the PhD grant under Sub-Action 8.2.2 Regional Innovation Strategies, Activity 8.2 Know-How Transfer, Priority VIII Regional Business Personnel of the Human Capital Operational Programme, co-funded from the EU resources within the European Social Fund as well as the state budget and the Lubuskie Voivodship.

\section{References}

1. Weiss, P.: L'hypothèse du champ moléculaire et la propriété ferromagnétique. J. Phys. 6, 661 (1907)

2. Kadanoff, L.P.: More is the same; phase transitions and mean field theories. J. Stat. Phys. 137, 777 (2009)

3. Landau, L.D.: Phys. Z. Sow. 11, 26545 (1937). English Translation: Collected Papers of Landau, L.D., ter Haar, D. (eds.) 193-215. Pergamon Press, Oxford (1965).

4. Stanley, H.E.: Introduction to Phase Transitions and Critical Phenomena. Oxford University Press, New York (1971)

5. Ginzburg, V.L., Landau, L.D., Eksp, Zh.: Teor. Fiz. 20, 1064 (1950)

6. Petschek, R., Metiu, H.: A computer simulation of the time-dependent Ginzburg-Landau model for spinodal decomposition. J. Chem. Phys. 79, 3443 (1983)

7. Bak, P., von Boehm, J.: Ising model with solitons, phasons, and "the devil's staircase". Phys. Rev. B. 21, $5297(1980)$

8. Opper, M., Saad, D.: Advanced Mean Field Methods, Theory and Practice. MIT Press, Cambridge (2001)

9. Carbonetto, P., de Freitas, N. :Conditional Mean Field in Advances in Neural Information Processing Systems 19 (eds.) Scholkope, B., Platt, J., Hofman, T., 201-209. Massachusetts Institute of Technology, Cambridge (2007).

10. Binder, K., Heermann, D.W.: Monte Carlo Simulation in Statistical Physics. An Introduction. Springer, Berlin (2010)

11. Netz, R.R., Berker, A.N.: Monte Carlo mean-field theory and frustrated systems in two and three dimensions. Phys. Rev. Lett. 66, 377 (1991)

12. Maier, W., Saupe, A.: Eine einfache molekulare theorie des nematischen kristallinflssigen Zustandes. Z. Naturforsch. Teil A 13, 564 (1958)

13. Picken, S.J., van der Zwaag, S., Northolt, M.G.: Molecular and macroscopic orientational order in aramid solutions: a model to explain the influence of some spinning parameters on the modulus of aramid yarns. Polymer 33, 2998 (1991)

14. Press, W.H., Teukolsky, S.A., Wetterling, W.T., Flannery, B.P.: Numerical Recipes. The Art of Scientific Computing, pp. 302-304. Cambridge University Press, Cambridge (2007)

15. Bray, A.J.: Theory of phase-ordering kinetics. Adv. Phys. 51, 481 (2002)

16. Bray, A.J., Rutenberg, A.D.: Growth laws for phase ordering. Phys. Rev. E 49, R27 (1994)

17. Lifshitz, I.M., Slyozov, V.V.: The kinetics of precipitation from supersaturated solid solutions. J. Phys. Chem. Solids 19, 35 (1961)

18. Nosé, S.: A molecular dynamics method for simulations in canonical ensemble. Mol. Phys. 52, 255 (1984)

19. Hoover, W.G.: Canonical dynamics: Equilibrium phase-space distributions. Phys. Rev. A 31, 1695 (1985) 